\title{
A MOVEMENT OPTIONS ANALYSIS SIMULATION TOOL FOR THE CANADIAN OPERATIONAL SUPPORT COMMAND
}

\author{
Patricia Moorhead \\ Centre for Operational Research and Analysis \\ Defence Research and Development Canada \\ 101 Colonel By Drive \\ Ottawa, ON K1A 0K2, CANADA
}

\author{
Gregory Campbell \\ Canadian Operational Support Command \\ 101 Colonel By Drive \\ Ottawa, ON K1A 0K2, CANADA
}

\begin{abstract}
This paper provides an overview of a movement options analysis simulation tool that has been developed for the Canadian Forces. The aim of the Movement Estimator Tool (MET) is to enable movement staff to quickly compare movement plan options and determine the "best" plan. Given a list of items to be moved, specifications for the lift assets that could be utilized, and a possible line of communication, the MET uses simulation to estimate the time and cost of the move for multiple possible movement plans, and provides a graphical representation of the cost/time tradeoff region. Movement staff can then decide upon the best course of action, taking into account issues such as lift asset availability, and time and budgetary constraints. The MET is used to analyze a hypothetical redeployment of the Canadian Forces' Disaster Assistance Response Team from Haiti.
\end{abstract}

\section{INTRODUCTION}

The Operational Support Movement (OS Mov) section of the Canadian Operational Support Command (CANOSCOM) is responsible for planning, scheduling, directing, coordinating and controlling the strategic movement of materiel and personnel in support of major continental and international Canadian Forces (CF) operations. This includes determining the integral and contracted strategic lift requirements for deployment, sustainment and redeployment, as well as estimating the cost of, and time needed to effect such movements.

One of the tools at the disposal of OS Mov has been a spreadsheet calculation aid which allows users to quickly estimate the number of airlift flights required to move personnel and cargo in support of an operation. The calculation aid considers only four fixed aircraft mixture options and does not calculate the time or cost associated with any of the options examined. While this application was useful, OS Mov required a more robust decision aid tool to assist with the determination of the "best" mix of air, sea and land transportation assets for a movement, based on time and cost considerations.

In the summer of 2009, CANOSCOM OS Mov requested development of the Movement Estimator Tool (MET). Given a list of items to be moved, specifications for the lift assets that could be utilized, and a possible line of communication (LOC - the geographical path from origin to destination that is to be taken), the MET estimates the time and cost of the move for multiple possible movement plans, and presents a graphical representation of the cost/time tradeoff region. OS Mov staff can then decide on the "best" plan taking into account issues such as lift asset availability, and time and budgetary constraints. Once a viable course of action is chosen, the detailed movement plan can be developed.

The MET is a stand-alone application, built in Visual Basic. As depicted in Figure 1, the tool has three main components: an application interface, a simulation module, and a calculation module. Users enter the necessary data via the application interface. This includes the movement load list, information 


\section{Moorhead and Campbell}

on the lift assets that could be used for the move, and the proposed LOC. Once the necessary data and planning factors have been entered, the simulation module is activated. The simulation module generates a series of movement scenarios, each of which is defined by a particular mixture of the possible lift assets. Each scenario is replicated 1000 times, with the source of variability coming from re-orderings of the items in the movement load list, and stochastically generated values for the maximum permissible payloads of the lift assets and the Canadian/American currency exchange rate. The scenario information is passed to the calculation module wherein the time and cost associated with the movement option is estimated. The results from the 1000 replications of each scenario option are automatically summarized and a graphical representation of the cost/time tradeoff region, which facilitates comparison of the various move plan options, is generated and displayed.

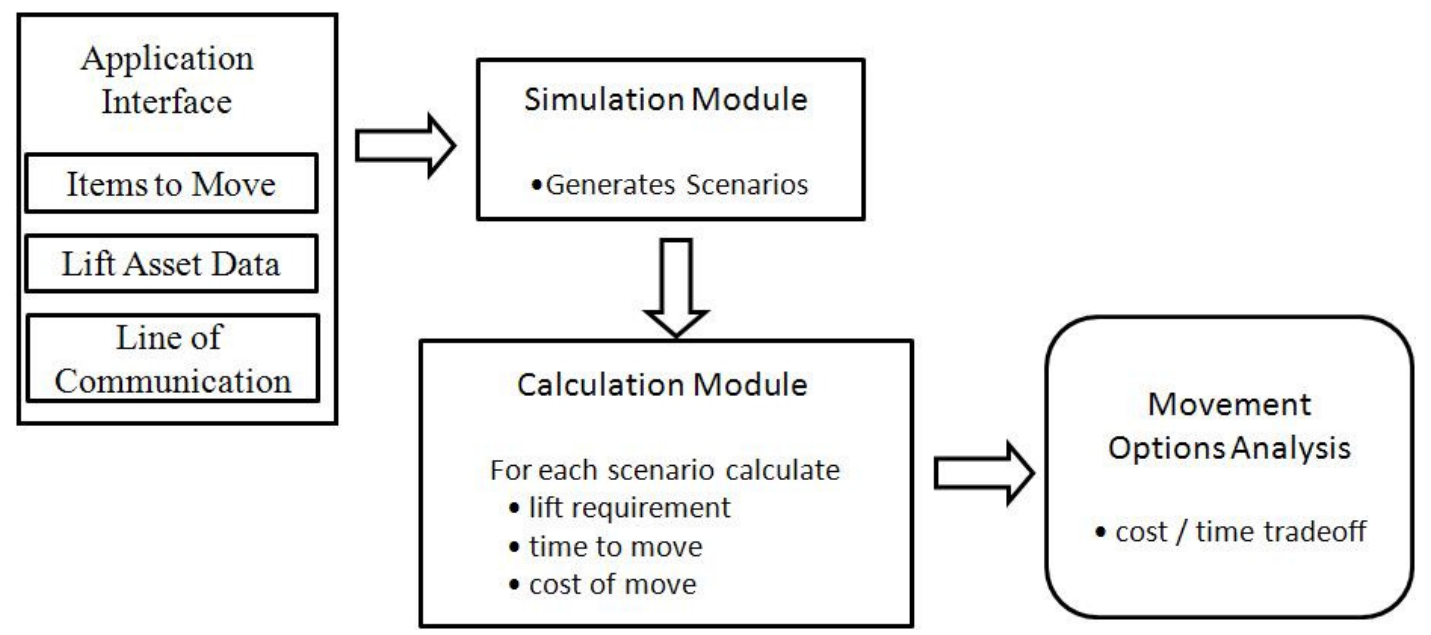

Figure 1: Components of the Movement Estimator Tool.

Development of Version 1.0 of the MET is complete. This initial version has some limitations in its functionality. A single mode of transportation, either airlift or sealift, is assumed and cross-loading between platforms during the move along the LOC is not currently supported. Version 2.0 of the tool will allow cross-loading between modes of transport along a single LOC, and also incorporate rail and road lift estimation capabilities.

The next section of this paper describes the process of setting up a simulation in the MET. The logic of the simulation and calculation modules are then described. In Section 5, the methodology utilized to analyze the simulation results is presented. An example application of the MET is provided in Section 6. Plans for further enhancing the functionality of the tool are discussed in the conclusion.

\section{SETTING UP A SIMULATION IN THE MET}

There are five steps to follow within the application prior to running a simulation. These are:

1. choose the mode of transportation for the movement;

2. create the movement load list;

3. choose a LOC;

4. declare the usage schedule for the strategic lift assets that could be used; and

5. enter/verify various lift planning factors. 


\subsection{Mode of Transportation}

In Version 1.0 of the MET the transportation options are restricted to airlift or sealift; rail and road lift options will be added in a future version of the application. Additionally, only one mode of transportation can be chosen; cross loading between airlift and sealift is not supported by the MET at this time.

\subsection{Movement Load List}

The MET provides users with a preformatted Excel spreadsheet that is to be populated with cargo information. For each type of item in the cargo list, the following information is required:

- movement priority (1 to 10 , with 1 being the highest);

- quantity of the item to be moved;

- item dimensions (length, width, height) and weight; and

- for sealift, which ship deck(s) the item can be loaded onto.

Linked to the MET is a database of common CF materiel. Selecting items from this database automatically adds them to the movement load list along with their dimension and weight information. Users then declare the movement priority, quantity, and ship deck(s) manually.

\subsection{Line of Communication}

The LOC is declared by selecting points on a global map. A minimum of two points (origin and destination) must be chosen. Once a path has been chosen, the MET automatically calculates the distance of the move using the haversine formula for great circle distance (Veness 2002).

\subsection{Lift Asset Usage Schedule}

The strategic lift assets to be considered for use during the move are selected from a list of integral and leased airlift and sealift assets commonly used by the CF. Users then declare the maximum number of departures by each type of aircraft (ship) that could occur, either per week or per month.

It should be noted that users do not declare how many instantiations of each asset type are to be used. The MET considers the number of trips per week/month; the actual number of airframes or ships to be used to complete these trips will be up to CANOSCOM OS Mov staff to decide.

\subsection{Other Planning Factors}

The final stage before simulating a movement plan is to declare values for the remaining planning factors, which can be grouped into three general categories: lift asset properties; loading constraints; and foreign exchange rates.

For each strategic lift asset type, the following planning factors are required by the MET.

- The cargo bay dimensions. For aircraft, only one cargo bay is assumed. For ships, the carrying capacity of each deck is declared separately.

- For sealift assets, the total lane meters per deck. One lane meter is an area $1 \mathrm{~m} \times 2.75 \mathrm{~m}$.

- The maximum permissible payload (MPL). As aircraft MPLs can vary with atmospheric conditions, the MET uses a triangular distribution to stochastically generate aircraft MPL values for each replication of a simulation. Users are requested to enter a lower limit, most likely value, and an upper limit for the MPL.

- The average speed of the asset.

- The cost to operate the asset. For aircraft this can be the cost per flight hour or the cost per return trip lease. For ships, users are asked to declare the hiring cost (\$US/day) and the cost of fuel (\$US/day). Since the price of fuel fluctuates, the MET will automatically generate a uniform distribution (with endpoints $\pm 10 \%$ of the entered cost) for the daily fuel cost for sealift; it is this stochastically generated value that will be used during simulations. 


\section{Moorhead and Campbell}

In addition to the strategic lift asset planning factors, there may be constraints on how the assets can be loaded with cargo. The MET requests users to declare values for the following influencing factors where relevant:

- inter-item distances (the spacing between items in the cargo bay);

- the maximum load restriction of the loading crane and/or loading ramp;

- the dimensions of the loading ramp;

- the dimensions and load restriction of the elevator between decks on sealift assets; and

- the number of layers of sea containers (up to 3) allowed on the decks of sealift assets.

Since most lease costs are provided in American dollars, the final planning factor required by the MET is the exchange rate between Canadian and American currencies. Users are requested to enter a minimum and maximum value; during simulations, the exchange rate is generated from a uniform distribution on this range.

All of the above mentioned planning factors are assigned validated initial values within the MET. Users can edit these values as required and save the changes.

\section{SIMULATION MODULE LOGIC}

The aim of the simulation module is to automatically cycle through all viable mixtures of airlift (or sealift) assets and to obtain cost and time estimates for moving the cargo using each mixture. In Version 1.0 of the MET, combinations are restricted to mixtures of at most two lift asset types of the same mode. For example, at most two types of aircraft can be used simultaneously to conduct an air move. Extending the simulation module logic to consider mixtures of three or more lift asset types of the same mode will be considered in a later version of the application.

For each mixture of lift assets considered, a total of 1000 replications are run. Across replications the sources of variability come from re-ordering of items on the movement load list, the MPLs of the airlift assets, and the values of certain cost factors. To ensure that the results are comparable across lift asset mixture options, the exact same 1000 replications must be used for each asset mixture scenario. This is accomplished by first looping through replications, and within each replication cycling through the lift asset mixture options. Figure 2 outlines the simulation module logic flow within each replication.

Step 1: For each asset type (aircraft or ship model) that is to be considered, generate an MPL value.

Step 2: Generate the currency exchange rate (\$Cdn to \$US).

Step 3: If sealift is to be used, generate the daily fuel cost for the sealift assets.

Step 4: Randomize the order of items in the movement load list.

Step 5: Generate an asset mixture (a pairing of two asset types).

Step 6: Send the scenario information to the calculator module, for estimation of the time and cost associated with movement of the listed cargo items by the asset mixture generated.

Step 7: Return to Step 5 and generate a new asset mixture. Loop between Steps 5 and 7 until all asset mixtures options have been examined.

Figure 2: Simulation module logic flow for each replication.

Within each individual replication, the first step is to generate an MPL value for each of the airlift asset types being considered for use. The MPL values are generated from the user-defined triangular distributions declared during simulation set up. The next step is to generate the foreign exchange rate from the uniform distribution declared by the user. If sealift is the mode of transportation for the move, the simulation module will generate a value for the daily cost of fuel for each sealift asset, using a uniform distribu- 


\section{Moorhead and Campbell}

tion. The lower and upper limits of the distribution are $90 \%$ and $110 \%$ of the planning value users declared previously.

The MET loads items into cargo bays according to priority (priority one items are loaded first), and within each priority group the items are loaded in the order they appear on the list. Altering this order can change the estimate of the number of trips required to move the cargo, due to differences in load configuration. Thus, the fourth step within each replication generated by the simulation module is to randomly reorder the items within each priority group listing.

At this stage the simulation module is ready to cycle through lift asset mixtures, calculating the cost and time to conduct the move with each specific asset combination. All possible pairings of airlift (sealift) types are considered in turn, with the number of weekly/monthly trips by aircraft (ships) of each type ranging from zero to the maximum number that was defined by the user.

Another feature of the MET is the ability to prioritize the utilization of lift assets. Prioritization determines the order in which the lift asset types are loaded. For example, if a weekly airlift utilization schedule has been declared, in each week the flights associated with the Priority 1 aircraft will be filled first, followed by the Priority 2 aircraft flights. This pattern is repeated every week until the move is complete.

For example, consider the situation wherein there are up to two Canadian owned CC-177 Globemaster III, one leased Antonov 124 (AN124) and one leased Ilyushin 76 (IL76) flights available per week. Table 1 shows the 14 prioritized mixture options that will be generated by the simulation module. For each of these unique mixtures of lift assets, the simulation module sends the mixture scenario and all other input data to the calculation module for estimation of the cost and time of the move.

Table 1: Prioritized mixture options for three airlift asset types.

\begin{tabular}{|c|c|c|}
\hline Mixture Option & Priority 1 Aircraft & Priority 2 Aircraft \\
\hline 1 & $1 \times$ CC-177 & nil \\
\hline 2 & $1 \times$ CC-177 & $1 \times$ AN124 \\
\hline 3 & $1 \times$ CC-177 & $1 \times$ IL76 \\
\hline 4 & $2 \times$ CC-177 & nil \\
\hline 5 & $2 \times$ CC-177 & $1 \times$ AN124 \\
\hline 6 & $2 \times$ CC-177 & $1 \times$ IL76 \\
\hline 7 & $1 \times$ AN124 & nil \\
\hline 8 & $1 \times$ AN124 & $1 \times$ CC-177 \\
\hline 9 & $1 \times$ AN124 & $2 \times$ CC-177 \\
\hline 10 & $1 \times$ AN124 & $1 \times$ IL76 \\
\hline 11 & $1 \times$ IL76 & nil \\
\hline 12 & $1 \times$ IL76 & $1 \times$ CC-177 \\
\hline 13 & $1 \times$ IL76 & $2 \times$ CC-177 \\
\hline 14 & $1 \times$ IL76 & $1 \times$ AN124 \\
\hline \multicolumn{2}{|c}{} \\
\hline
\end{tabular}

This entire process (from generating MPL values to estimating the cost and time of a move plan) is repeated 1000 times, thus generating 1000 cost/time data points for each unique mixture of strategic lift assets considered.

\section{CALCULATION MODULE LOGIC}

Given a movement plan scenario (a list of items to move, a lift asset mixture to use, a LOC and values for the planning factors), the calculation module estimates the cost and time of the planned move.

Recall that users are required to declare a lift asset usage schedule when setting up a simulation. This dictates the maximum number of departures, per week or month, which can be made by each asset type. By counting the number of repeats of this usage pattern required to complete the move, the calculation 


\section{Moorhead and Campbell}

module estimates how many individual trips will have to be made by each asset type as well as the time the move will take. To determine the number of weekly/monthly schedule repeats required, the calculation module emulates loading items, in the order they appear on the movement load list, into lift assets in the order dictated by the asset usage schedule and priorities.

For example, consider mixture option \#5 from Table 1, wherein there are two CC-177 flights and one AN124 flight per week, with the CC-177 being the priority one asset. In the first week of the move, the MET will load cargo items into the first CC-177 flight. When the first CC-177 flight is full, the second CC-177 flight will be loaded. Only after both CC-177 flights are full will the single AN124 flight for that week be loaded with items. At this stage one week is considered to have passed, with two CC-177 and one AN124 flight. This process is repeated until all cargo items have been moved; the total number of flights by each aircraft type and the total number of weeks required are recorded.

As previously mentioned, the movement load list is ordered by priority, with priority one items appearing first. Cargo items are loaded onto lift assets in the order in which they appear on this list, and the general philosophy is to fill each lift asset as much as possible. Following the example above, the first item on the movement load list will be loaded onto the first CC-177 flight, if the item fits. Both fit by volume and weight are assessed.

There are three steps to assessing item fit. First, each item is assessed to determine whether or not on its own it would cause bulk-out or weigh-out of the lift asset. Items failing this test cannot be moved by this asset type. Second, the percentages of the lift asset's carrying capacity, by area and weight, occupied by the item are calculated. Let $L_{a}$ and $W_{a}$ be the length and width of the cargo bay, and $M_{a}$ the MPL of the lift asset. Let $L, W$ and $M$ be the length, width and weight of the item, respectively. If the inter-item distances in the cargo bay are $d_{l}$ (lengthwise) and $d_{w}$ (widthwise), then the percentages of the lift asset's carrying capacity occupied by the item are given by

$$
\% C C_{\text {Area }}=\left\{\left\lfloor\frac{L_{a}-d_{l}}{L+d_{l}}\right\rfloor *\left\lfloor\frac{W_{a}-d_{w}}{W+d_{w}}\right\rfloor\right\}^{-1} \quad \text { and } \% C C_{\text {Weight }}=\left\{\left\lfloor\frac{M_{a}}{M}\right\rfloor\right\}^{-1},
$$

where $\lfloor\cdot\rfloor$ is the floor operator. The space remaining on the lift asset by area and weight will be one minus each of these quantities respectively.

The third step is to compare the $\% C C_{\text {Area }}$ and $\% C C_{\text {Weight }}$ occupied by the new item to the available capacity remaining in the lift asset. If the item will not fit by either area or weight, the MET will search the load list in sequential order to see if any items lower down on the list will fit in the remaining space. This process is repeated until no more items will fit into the first CC-177 flight. The MET then begins to fill the second CC-177 flight. The first item to be loaded onto this flight will be the first item on the movement load list that fits and has not yet been loaded.

When determining whether or not an individual item fits into the available space on a lift asset, additional factors such as crane, elevator and loading ramp dimensions, and load carrying constraints are taken into consideration where relevant. Items that cannot be loaded onto any of the asset types chosen are brought to the attention of the user. It should be noted that the MET does not replace the role of the loadmaster; the application does not take into consideration load balancing issues when assigning items to lift assets. It may be the case that the set of items assigned to a given lift asset trip may form a balanceable load, but this must not be assumed.

The weekly/monthly usage schedule of the lift assets will be repeated until all items have been moved. The number of pattern repeats provides the time estimate; ten repeats of a weekly pattern indicate that ten weeks will be required to complete the move. Multiplying the number of trips per week/month for each lift asset type by the number of schedule pattern repeats yields the total number of trips required by each asset type.

The total cost of a move is the sum of the costs incurred by each Canadian owned and leased lift asset that was utilized. Let $T_{i}$ be the number of trips taken by asset type $i$. For Canadian owned lift assets, de- 


\section{Moorhead and Campbell}

note asset speed and hourly operating cost by $v_{i}$ and $r_{i}$ respectively. For leased lift assets, contracts typically stipulate a fixed cost for a single use of the asset, whether it is a one-way or return trip, commencing in Canada or another nation. Let $R_{i}$ denote the lease cost of non-integral lift asset type $i$. If $D$ is the oneway trip distance to be travelled by the lift assets, the total cost of the movement is given by

$$
\text { Total Cost }=\sum_{i \in C d n} T_{i} * \frac{2 D}{v_{i}} * r_{i}+\sum_{i \in \text { Leased }} T_{i} * R_{i}
$$

The one-way distance is doubled for Canadian strategic lift assets as it is assumed they will always begin and end their journey in Canada.

Validation and verification of the detailed calculation module algorithms have been conducted jointly by operational research analysts and CANOSCOM OS Mov staff. Additionally, several test cases were examined and the results produced by the MET compared to those obtained from previous tools at the disposal of OS Mov staff and via subject matter expertise. In all cases the MET produced valid results. OS Mov began using a beta version of the calculation module in November 2009 to support several movement plan analyses. The MET was used extensively for the planning of Canada's humanitarian assistance mission to Haiti in early 2010. Based on these experiences, the MET has been approved by CANOSCOM OS Mov chain of command and is being adopted as a primary movement planning tool for the section.

\section{PRESENTING SIMULATION RESULTS}

For each unique combination of strategic lift assets considered for a move, the MET generates 1000 cost/time estimates that take into consideration the variability in MPLs (due to changes in atmospheric conditions), currency exchange rates and fuel costs, as well as the impact of load patterns on the number of trips that may be required. The dominant driver in determining both the cost and time of a move will be the set of MPL values - the less (more) a lift asset can carry, the greater (fewer) the number of trips are necessary, and the cost of the move increases (decreases).

Two types of graphs are produced by the MET. The first is a time cumulative frequency plot, which indicates the reliability of being able to complete the move within a certain number of weeks/months. For each lift asset mixture, the number of times within the 1000 replications that each movement time period is observed is tabulated and the cumulative total is plotted against the observed time values. From this graph, movers can readily assess the reliability of completing a move within a given time frame.

The second graph presents the cost/time trade-off region. For each observed time value in the 1000 replications for a given asset mixture, the $90^{\text {th }}$ percentile of the set of corresponding cost values is extracted. This particular percentile was chosen so as to provide conservative estimates of the cost of a move. The curve defined by these data points is taken as being representative of the cost/time tradeoff region for that asset mixture.

The following section provides an example of how these two types of graphs may look and be interpreted.

\section{EXAMPLE APPLICATION}

As previously mentioned, a prototype of the MET was used to assist with planning of Operation HESTIA, Canada's humanitarian assistance mission to Haiti in early 2010. As part of this mission, the Disaster Assistance Response Team (DART) was deployed. To demonstrate the utility of the MET, an example redeployment of DART equipment from Port-au-Prince, Haiti to Trenton, Ontario will be used.

A DART equipment list was provided by CANOSCOM OS Mov staff, and consists of 50+ items such as trailers, heavy lift vehicles, logistic support vehicles, generators and reverse osmosis water purification units. As priority ranks for the items were not provided, priority levels of one to three were randomly assigned to the items on the movement load list. 


\section{Moorhead and Campbell}

During the actual redeployment from Haiti, both air and sea transport were utilized. As Version 1.0 of the MET accommodates only one mode of strategic lift at a time, the (artificial) assumption that all items are to be redeployed via direct airlift was made. Three aircraft types were considered: CC-177s, AN124s and IL76s. A weekly usage schedule was assumed, with up to two CC-177 flights per week but only one each of the AN124 and IL76. The set of 14 possible prioritized airlift mixture pairs are the same as those shown in Table 1.

For the purposes of this demonstration, artificial rather than actual cost data was utilized. It was assumed that one return trip between Trenton and Port-au-Prince with a Canadian owned CC-177 costs \$170K (Cdn). Lease costs were taken to be \$1M (US) and \$300K (US) per trip, for the AN124 and IL76 respectively. During simulation, a uniform distribution on the interval $[0.93,1.00]$ was used to generate Canadian to American dollar exchange rate values, which were then applied to the lease costs.

The triangular distributions chosen for the aircraft MPLs are shown in Table 2. The minimum and maximum values are based on CF planning factors. The mode values aim to reflect the most likely MPL for flights out of Port-au-Prince.

Table 2: Triangular distributions for aircraft MPLs.

\begin{tabular}{|c|c|c|c|}
\hline Aircraft & MPL Minimum & MPL Mode & MPL Maximum \\
\hline CC177 & $20,000 \mathrm{~kg}$ & $40,800 \mathrm{~kg}$ & $77,500 \mathrm{~kg}$ \\
\hline AN124 & $25,000 \mathrm{~kg}$ & $55,000 \mathrm{~kg}$ & $80,000 \mathrm{~kg}$ \\
\hline IL76 & $15,000 \mathrm{~kg}$ & $25,000 \mathrm{~kg}$ & $35,000 \mathrm{~kg}$ \\
\hline
\end{tabular}

The above mentioned data and planning factor information was entered into the MET. A total of 1000 replications were run; within each of these, the cost and time of the move was calculated for each of the 14 prioritized airlift mixture options. Thus 1000 cost/time data points were generated for each airlift mixture option, based on varying load configurations, MPLs and exchange rate. Not all of the cost values generated were valid as some items did not fit in all aircraft flights, either due to dimensional constraints or MPL values that were too low, resulting in incomplete moves. All such data points were removed prior to analyzing the results. For example, the IL76 was incapable of moving all of the DART equipment due to item bulk-out issues, so asset mixture option \#11 (IL76 only) was removed from the analysis.

Suppose the DART redeployment is be completed in 15 weeks or less, with a reliability level of $80 \%$ or higher (i.e., the risk of failing to meet the time constraint is to be no more than 20\%). An examination of time cumulative frequency graphs for the thirteen prioritized asset mixture options shows that only nine meet these criteria. In Figure 3 we see a comparison of the time cumulative frequency curves for these nine options.

Using a mixture of one IL76 and one AN124 flight per week, the move was completed within the desired 15 weeks in approximately $85 \%$ of the simulation replications. The other airlift mixtures always completed the move in 14 weeks or less. The fastest move was accomplished using a mixture of two CC177 flights and one AN124 flight per week. With this airlift combination, the move was completed within seven weeks with $88 \%$ reliability. 


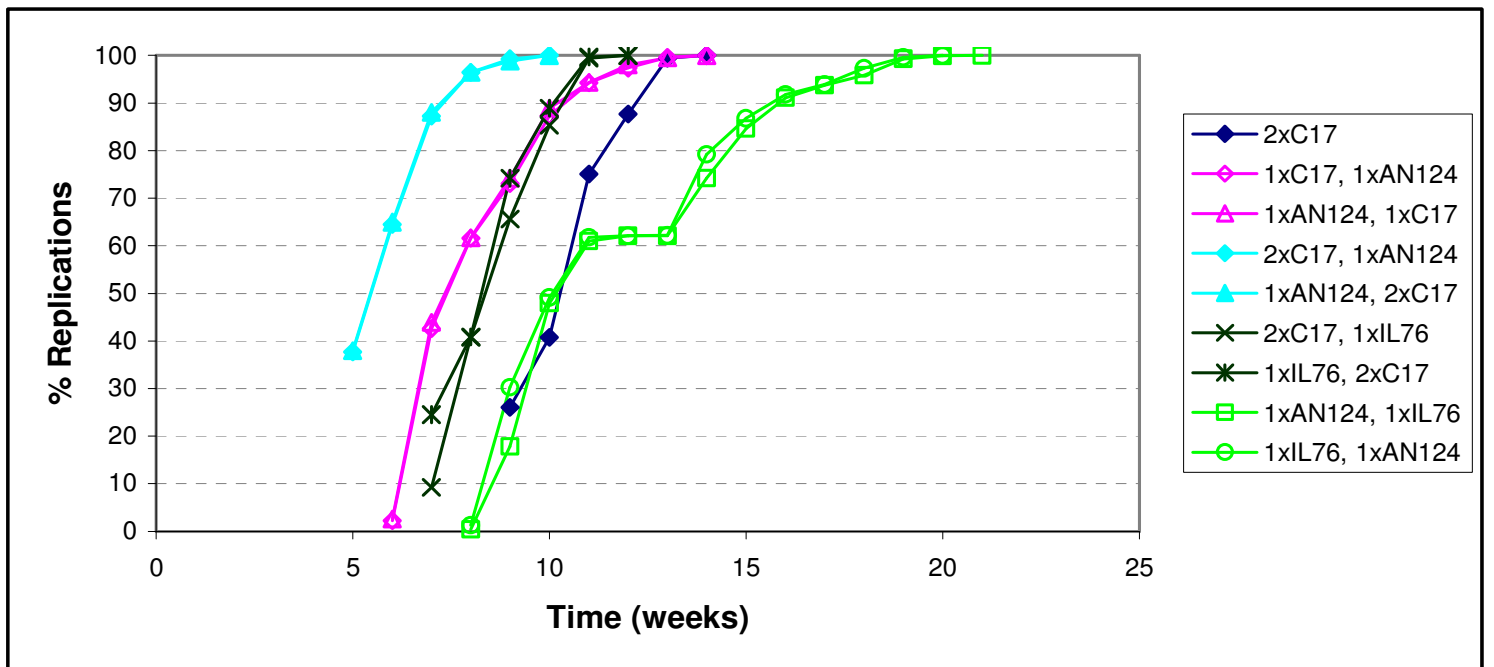

Figure 3: Time cumulative frequency curves for airlift mixtures satisfying the redeployment criteria.

The cost/time tradeoff region for these nine airlift mixture options is shown in Figure 4. On a simple cost comparison basis, the least expensive option was two CC-177 flights per week, while the most expensive was the AN124 and IL76 combination. This is due to the fact that leased aircraft cost more per trip than Canadian owned aircraft.

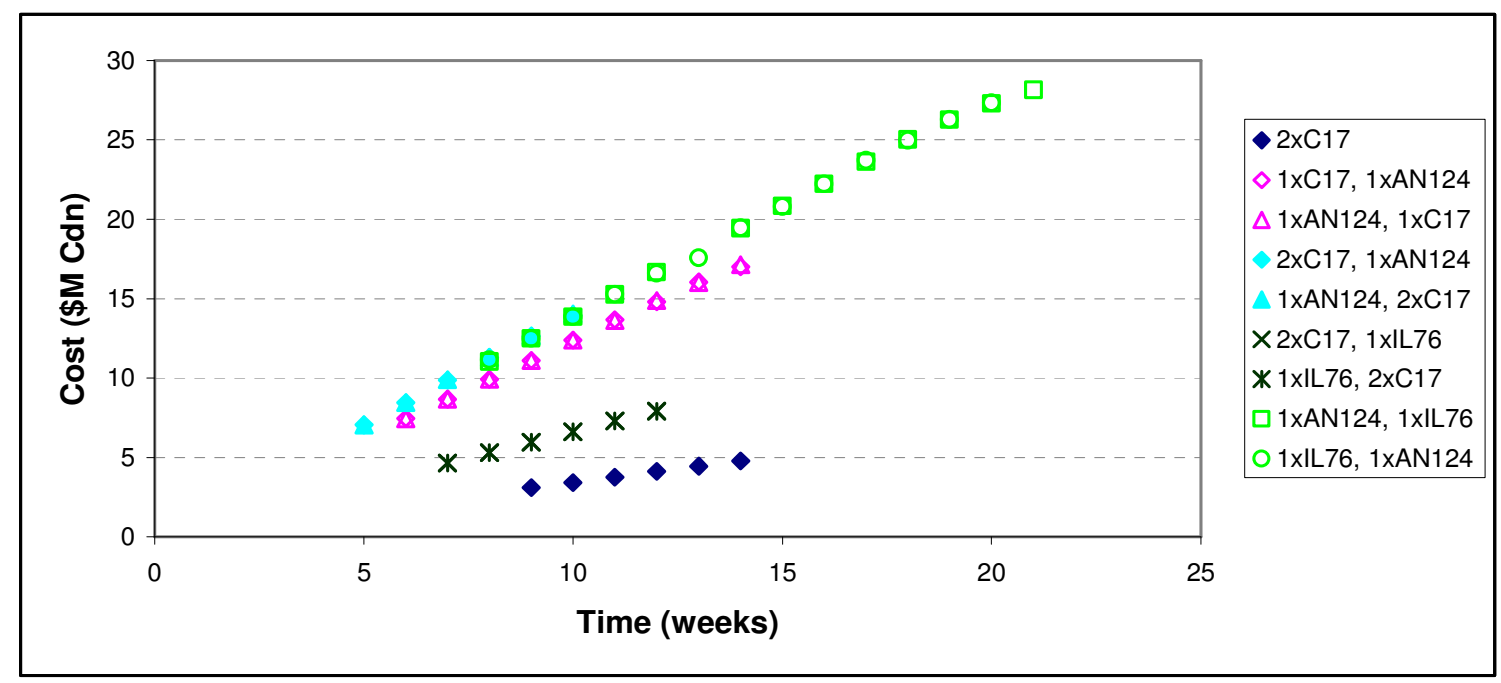

Figure 4: Cost/time tradeoff region for airlift mixtures satisfying the redeployment criteria.

By combining the results of Figures 3 and 4, movement staff can weigh the various movement plan options against each other. The time requirement and cost estimate to obtain the desired minimum $80 \%$ reliability for each of the viable airlift mixture options are as follows:

- 2 x CC-177 - 12 weeks, $\$ 4.1 \mathrm{M}$;

- 2 x CC-177 and 1 x IL76 - 10 weeks, \$6.6M;

- 2 x CC-177 and 1 x AN124 - 7 weeks, $\$ 9.9 \mathrm{M}$;

- 1 x CC-177 and 1 x AN124 - 10 weeks, $\$ 12.4 \mathrm{M}$; and

- 1 x AN124 and 1 x IL76 - 15 weeks, \$20.8M. 


\section{Moorhead and Campbell}

If this DART redeployment scenario was a real situation, CANOSCOM OS Mov staff would utilize these results, to inform their decision as to the "best" asset mixture to complete the move. Should cost be the primary factor, the preferred option would be to use two CC-177 flights per week. If there is flexibility in the budget, additional investment can reduce the time required to meet the desired $80 \%$ reliability level. For an additional $\$ 2.5 \mathrm{M}$, the movement timeframe can be reduced by two weeks by adding a single IL76 flight to the two weekly CC-177 flights. Alternately, replacing the additional IL76 flight with that of an AN124 will cost \$5.8M more than two CC-177 flights alone, but yields an overall reduction of five weeks in the time needed to achieve $80 \%$ reliability.

\section{CONCLUSION}

This paper provided an overview of the MET, a movement options analysis simulation tool that has been developed for CANOSCOM OS Mov. The application enables movement staff to quickly compare move plans and select the "best" plan, based on time and cost considerations. The main inputs to the MET are: the movement load list; specifications for the lift assets that could be utilized; and a possible LOC. Based on this information, the MET simulates the move for multiple possible movement plans, and estimates the time and cost associated with each plan. The cost/time tradeoff regions for each option are presented graphically for easy comparison. OS Mov staff can then decide upon the preferred course of action, taking into account issues such as asset availability, and time and budgetary constraints. An example application, redeployment of DART equipment from Haiti, was presented.

Version 1.0 of the MET has been used extensively by CANOSCOM OS Mov staff, for humanitarian mission planning (e.g., Op HESTIA) and examining the pending redeployment of Canadian Forces from Afghanistan in 2011. Development of Version 2.0 of the tool is currently underway. In this phase crossloading of cargo between modes of transportation along the LOC will be incorporated. The current restriction on asset combinations to at most pairs of assets will be removed, allowing for combinations of three or more lift assets of the same mode to be considered simultaneously. Finally, road and rail options for the mode of transportation will be incorporated into the tool.

\section{REFERENCES}

Veness, C. 2002. Calculate distance, bearing and more between Latitude/Longitude points. Movable Type Ltd. Available via <http://www.movable-type.co.uk/scripts/latlong.html> [accessed October 5, 2009].

\section{AUTHOR BIOGRAPHIES}

PATRICIA MOORHEAD has a B.Sc. in Mathematics and Statistics, and a M.Sc. in Statistics from the University of Guelph. Since 2002, she has been employed as a Defence Scientist with Defence Research and Development Canada - Centre for Operational Research and Analysis. She is currently posted to the Canadian Operational Support Command Headquarters, and is conducting operational research studies on personnel, logistics and readiness issues related to joint operational support. Her e-mail address is

<patricia.moorheadedrdc-rddc.gc.ca>.

GREGORY CAMPBELL has a B.Sc. in Biochemistry, a diploma in healthcare/OTC product chemistry and formulation, and a Master degree in business administration from the Royal Military College of Canada. He is a reserve infantry officer employed as an Operational Research Analyst with the Canadian Operational Support Command. His research interests include analytical decision support techniques, quantitative methods, statistics, and simulation in support of solving military problems. He has served as a military officer since 1994 and seeks to further his career as an Operational Research Scientist. His e-mail address is <Gregory. campbelleforces.gc.ca>. 